\title{
Weekends as social distancing and their effect on the spread of influenza
}

\author{
Philip C. Cooley ${ }^{1}$ Sarah M. Bartsch ${ }^{2}$. \\ Shawn T. Brown ${ }^{3}$ - William D. Wheaton ${ }^{4}$. \\ Diane K. Wagener ${ }^{5} \cdot$ Bruce Y. Lee $^{2}$
}

Published online: 5 October 2015

(c) Springer Science+Business Media New York 2015

\begin{abstract}
Many published influenza models treat each simulation day as a weekday and do not distinguish weekend days. Consequently, the weekend effect on influenza transmission has not been fully explored. To assess whether distinguishing between weekday and weekend transmissions in simulation models of flu-like infectious disease models matters, this study uses an agent-based model of the Chicago, Illinois metropolitan area. Our study assesses whether including weekend effects is offset by increases in weekend contact patterns and if implementing 3-day weekends dampens disease transmission enough to warrant its use as a containment strategy. Results indicate that ignoring weekend behaviors without incorporating increases in community-based non-school contacts (i.e., compensatory behaviors) causes the peak case incidence day to occur 7 days earlier and can reduce the peak attack rate by as much as $60 \%$. These results are sensitive to the proportion of symptomatic cases that are assumed to remain at home until they recover. The 3-day weekend intervention has interesting possibilities, but the benefits may only be effective for mild epidemics. However, a 3-day weekend for schools would be less detrimental to the educational process than sustained permanent closing because student and teacher contact is maintained throughout the epidemic period. Also, a 4-day school and work week may be more easily accommodated by many types of schools and businesses.
\end{abstract}

Philip C. Cooley

pcc@rti.org

1 Research Computing Division, RTI International, Research Triangle Park, NC 27709, USA

2 Public Health Computational and Operations Research, Johns Hopkins University, Baltimore, MD 21218, USA

3 Pittsburgh Supercomputing Center, Carnegie Mellon University, Pittsburgh, PA 15213, USA

4 Geospatial Science and Technology Program, RTI International, Research Triangle Park, NC 27709, USA

5 Genomics, Statistical Genetics and Environmental Research Program RTI International (Retired), San Diego, CA 25628, USA 
On the other hand, an additional day per week of school closure could result in substantial societal costs, with lost productivity and child care costs outweighing the savings of preventing influenza cases.

Keywords Influenza - Influenza transmission · Epidemics · Weekends · Intervention strategy $\cdot$ Agent-based model

\section{Introduction}

Although studies have explored the effects of weekends on securities prices (Chen and Singal 2003), vehicle emissions (Marr and Harley 2002), atmospheric dynamics (Atkinson-Palombo et al. 2006), and medical outcomes (Albright et al. 2009; Spiers and Guntheroth 1999), only Mao (2011) has explored the effect of weekends on the spread of influenza, either in a seasonal or novel strain epidemic. With workplaces, school, and many other locations of higher transmission closed, one may expect influenza transmission (or other infectious diseases transmitted via casual contact) to decrease during weekends. Indeed, some studies have shown that influenza transmission decreases over holidays as well as weekends (Cauchemez et al. 2008; Hens et al. 2009; Eames et al. 2010, 2011, 2012).

Mao (2011) used an agent-based model (ABM) with many design features similar to the one used for this study. However, the study region is dissimilar, with a much smaller study population of just under 1 million, while the Chicago area population is over 6 million. Mao used the model to assess a number of temporary school closing options that included closing for more than 2 days a week. We focused on the option of adding one more day to the 2-day weekend, not distinguishing between a Friday or a Monday closure, because we consider this option as a practical alternative to temporary school closure. Based on the school closure response to the 2009 H1N1 epidemic in Pennsylvania school officials are reluctant to close schools longer than one or 2 weeks. Our study also extends the results reported in Mao (2011) by providing an economic analysis of the 3-day weekend policy and assessing how long to maintain the policy before reverting back to the 2-day status quo.

School closings reduce student contacts, an intervention strategy often considered during epidemics. Araz et al. (2012) report that school closures can significantly reduce the total number of influenza cases. However, Lee et al. (2010c) demonstrated schools may need to be closed for lengthy periods (e.g., throughout the duration of the epidemic) to be effective. In fact, school closures that do not extend throughout the course of an epidemic may actually worsen it. Reopening schools refuels the epidemic by releasing a large number of susceptible children back into the transmission pool. Lempel et al. (2009) characterized the substantial financial cost of closing schools to mitigate an epidemic. Brown et al. (2011) determined that if school closure had been widely used as a mitigation strategy for the 2009 H1N1 epidemic in Pennsylvania, the cost may have far outweighed the potential costsavings from reducing the number influenza cases.

These observations raise the possibility that extending the weekend from 2 to 3 days could be a cost-effective control strategy and may not drastically effect 
society as lengthy school closures do. But, because many existing published influenza models treat each simulation day as a weekday and do not distinguish weekends, this effect on influenza transmission has not been fully explored.

One argument for not considering extended weekends as an important mechanism and not including it in models of influenza spread is the assumption that students engage in compensatory behaviors on the weekends that make up for the school contact (Cauchemez et al. 2008). The compensatory behaviors referenced include additional trips to the mall, the grocery store, etc., and "hanging out" with friends.

A survey by McCaw et al. (2010) indicated that an individual has two to four more personal contacts during weekends than weekdays. However, a study of university students found that individual contacts per day declined by $27 \%$ during weekends (Edmunds et al. 2006). This is clearly a parameter that requires sensitivity investigation.

This study uses an ABM of the Chicago, Illinois metropolitan area to assess if including weekend effects in simulation models of infectious disease transmissions matters. It also addresses whether including weekend effects is offset by increases in weekend contact patterns. Finally, it assesses whether extending the weekend to 3 days has a sufficient effect on disease transmission to warrant support as an intervention mechanism. The influenza natural history parameters used in this model were developed by Ferguson et al. (2005). They characterize how infected persons pass from pathogen exposure to the appearance of symptoms within mean 1.48 days infection, followed by an infectious period that produces a generation time of 2.6 days, after which they recover and are immune to repeated infection or die. The U.S. Centers for Disease Control and Prevention (CDC) reported the generation time of H1N1 2009 influenza as 2.0-3.1 days (Centers for Disease Control and Prevention 2009).

Also, Cauchemez et al. (2009) report the mean time between the onset of symptoms in a case patient and the onset of symptoms in the household contacts infected by that individual to be 2.6 days. To accommodate prior immunity to H1N1, we used age-specific estimates from a cross-sectional serological study in England (Miller et al. 2010).

\section{Materials and methods}

\subsection{Chicago: Cook and DuPage county study area}

Chicago is the county seat of Cook county, with a small portion extending into DuPage county. Collectively, the two counties contain a metropolitan area of $6,184,869$ residents, with adjoining suburban and rural areas. The region's population is $78 \%$ urban white and workplace density centers exist in both counties (U.S. Census Bureau 2009).

A synthetic agent database was developed to represent the human population of the study region (Wheaton et al. 2009). In this ABM, agents represent individual persons. Complex, large-scale social systems are simulated by assigning behaviors and activities to agents within the population, then allowing the agents to interact with each other and the environment. 
The synthesized agent database was adapted and extended from a method originally developed by Beckman et al. (1996) that employs the U.S. Census Bureau's Public Use Microdata files and census-aggregated data to generate synthesized, geospatially explicit human agents that represent actual populations when aggregated (Wheaton et al. 2009). Each agent is assigned to a household with other agents. Similarly, agents are individually assigned to schools and workplaces with other agents. After generating a U.S. population of 105,480,101 households containing 273,624,650 people (2000 Census), data for Cook and DuPage counties was extracted.

\subsection{Model description}

In each individual agent, disease progresses through an underlying susceptibleexposed-infectious-recovered (SEIR) disease model. At the start of each simulation run, individuals who have already been vaccinated or infected (recently or remotely) are classified as recovered (R), and therefore immune to, subsequent infection. An H1N1 vaccine was not available at the start of the epidemic so we assumed no one in the simulation was protected except those subjects with prior immunity. All other individuals were initially susceptible $(S)$ to influenza. At the start of each simulation run, 100 randomly chosen agents were infected to generate the epidemic. Every susceptible individual who contacted an infectious individual had a probability of contracting influenza (Longini et al. 2005; Halloran et al. 2008). Newly infected agents then progressively move into the exposed (E) state, in which they remain for the duration of the disease's incubation period. They then move to the infectious (I) state, in which the agent can infect others. In the simulation, the length of time in a given state is determined by randomly selecting variants from an incubation period and from infectious period distributions.

Two-thirds of infectious patients manifest symptoms; the remaining third are asymptomatic but are able to transmit disease [Writing Committee of the WHO Consultation on Clinical Aspects of Epidemic (H1N1) 2009]. The presence of symptoms is associated with higher transmissibility; typically, symptomatic infections are assumed to be twice as infectious as asymptomatic infections. However, one recent study estimated the infectious rate to be 3-12 times higher for symptomatic infections (Van Kerckhove et al. 2013). Our model assumes the lower value, so our symptomatic agents are three times as infectious as asymptomatic agents. According to data from the U.S. Bureau of Labor Statistics $(2013,2014)$, $34 \%$ of the workforce works on weekends and $63 \%$ of the adult population works. Because a portion of the $63 \%$ working population is unemployed, we estimated that $21 \%$ of all adults work on weekends. Thus, our model also assumes that $21 \%$ of randomly selected adults work on weekends (consistent with Bonne 2003) and that $90 \%$ of sick students and workers, or agents with symptomatic disease, remain at home without generating community-level contacts unless they see a doctor. Previous studies explore the lower rates of withdrawal to the home (Cooley et al. 2010, 2011, Lee et al. 2010a, 2010b; Rothberg and Rose 2005). The sensitivity of changes to these assumptions and their effect on simulated epidemics has been examined (Cooley et al. 2011). However, a recent study reported significantly fewer 
contacts among people with influenza symptoms; consequently, this study assumes the higher withdrawal rate resulting in reduced social mixing patterns (Van Kerckhove et al. 2013). The ABM used for this study also tracks features similar to those defined by Ferguson et al. (2006) and Germann et al. (2006), including age, sex, occupation, household location, household membership, school assignment of students and teachers, work location assignment of employed adults, work status as employed or unemployed, and disease status.

Our model assigned 1,185,062 school-aged children to 2103 school locations. The ArcGIS Business Analyst (ESRI, Redlands, CA) identified 162,648 businesses with total employment of 2,504,324 in Cook and DuPage counties. We assigned $2,584,324$ working adults to one of the workplaces by using the census 2000 special tabulation: census tract of work by census tract of residence (STP 64) database. This later data source supports an accurate representation of commuting patterns. Also, based on prior work (Cooley et al. 2011), we determined that incorporating public transportation into our model introduced extra complexity while contributing little to influenza transmission levels. We therefore decided not to model transportation; thus, agents move instantaneously between activities.

A fuller discussion of the assignment process is described elsewhere (Cajka et al. 2010).

Note that alternative models to our social network model exist that are driven by activity networks compiled from surveys of activities that occur throughout the day on a sample of representative persons. The sample traits are then synthesized and assigned to the entire simulation population. This approach legitimately argues that they represent a wider and more heterogeneous distribution of agent behaviors. However, these attributes are undermined because they also represent all days in a week as identical (our models distinguish between weekdays and weekends).

Furthermore, the Halloran et al. study (2008), compared three models of the Chicago metropolitan area. One of these (Episims) was an activity-based network model. The other two were social network models. While each generated different infection curves, all of the models ranked the value of the simulated intervention policies in the same order. By this policy evaluation criteria, there was no difference between the two model approaches.

\subsection{Model parameters and social network structure}

The ABM infection transmission probabilities are a product of the probability of daily contacts of sufficient duration and, given a contact occurs, the probability of transmission. The contact probabilities (Table 1) were obtained from two related studies by Longini et al. (2005) and Germann et al. (2006). Both sources are derived from data on the 1957-1958 Asian influenza epidemic. The contact probabilities in Table 1 depend on the age of both the infectious and susceptible persons and represent the likelihood of these two individuals having a daily contact of sufficient duration and closeness to transmit influenza.

The 6,184,869 individuals who are the region's household population are the model's circulating agents. The agents' locations are tracked and those who interact with other agents in close proximity are potential flu transmitters. For example, an 
Table 1 Agent-based model and daily person-to-person contact parameter values within contact groups

\begin{tabular}{llll}
\hline Contact group & Type of infected agent & Type of susceptible agent & $\begin{array}{l}\text { Probability of } \\
\text { daily contact }\end{array}$ \\
\hline Household & Adult & Adult & 0.4 \\
Household & Child & Adult & 0.3 \\
Household & Adult & Child & 0.3 \\
Household & Child & Child & 0.6 \\
School & Elementary student grades k-6 & Elementary student grades k-6 & 0.0435 \\
School & Middle Student grades 7-9 & Middle student grades 7-9 & 0.0375 \\
School & High student grades 10-12 & High student grades 10-12 & 0.0315 \\
Workplace & Adult & Adult & 0.0575 \\
Hospital & HCW ${ }^{1}$ & HCW & 0.0575 \\
Hospital & HCW & Patient & 0.01 \\
Hospital & Patient & HCW & 0.01 \\
Neighborhood & All & Child & 0.0000145 \\
Neighborhood & All & Adult & Child \\
Community & All & Adult & 0.000725 \\
Community & All & & 0.00003175 \\
\hline
\end{tabular}

\footnotetext{
1 Health care worker
}

agent interacts daily with family members; nonfamily members sharing a household interact with each other less than daily but at least four times a week.

Also, each student or worker also has a random probability of interacting with people in other classrooms or offices. There are 2103 schools in the region educating 1,185,062 students, and there are 162,245 workplaces (including hospitals and clinics) employing 2,584,324 workers. In schools and workplaces, each individual contacts the same set of persons each day, although the number of contacts vary by day. Workers in small firms have repeated contacts with the same people daily and all agents, including students, interact in the community daily including weekends, with student-community interactions increasing on weekends.

\subsection{Model calibration}

We calibrated our model using data from the H1N1 epidemic as reported by Cauchemez et al. (2009). These data are summarized in Table 2 and were based on an analysis of a pandemic influenza outbreak in an elementary school that spread to a rural community.

Calibration involved targeting an epidemic with a $16 \%$ illness infection attack rate (AR). This is based on the rate reported by Riley et al. (2011) for the 2009 H1N1 epidemic in Hong Kong.

The number of transmissible contacts are estimated endogenously to reproduce the proportion of infections that occur by place i.e. the observed distributions of infections by place represented in Table 2 . This process accounts for prior immunity by age and estimates the following contact rates: 
Table 2 Quantification of H1N1 transmission occurrences

See Cauchemez et al. (2009), Fig. 4

\begin{tabular}{lll}
\hline Source infected & Where infected & Infected (\%) \\
\hline Students & Household & 13 \\
& Class & 30 \\
& Grade & 22 \\
& School & 24 \\
& Community & 11 \\
Household members & Household & 30 \\
& Community & 70 \\
\hline
\end{tabular}

- students at school,

- students at schools within classrooms,

- adults in the workplace,

- students in the community,

- adults in the community

- adult to adult in the household,

- child to child in the household,

- adult to child in the household, and

- child to adult in the household.

The endogenously estimated daily contact rates are placed into a standard SEIR framework used to describe infectious disease models. The social network assumptions are straightforward. Students mix with other students assigned to their school as well as their classroom. Workers mix with workers assigned to their workplace. Household dwellers mix with other persons living within the household and everyone mixes with other people in the community such that the probability of a contact is inversely related to the distance between the infected person's household location and a random number of persons living within the simulated region (also estimated endogenously).

Under the above constraints, the estimated contact patterns are designed to reproduce an epidemic similar to the H1N1 flu epidemic of 2009, with a sickness (symptomatic) attack rate of $16 \%$, a total infection (asymptomatic plus symptomatic) rate of $24 \%$, and a basic reproductive rate $\left(\mathrm{R}_{0}\right)$ of 1.2.2.5 FluEcon: influenza economic model.

The number of symptomatic cases and the number of people missing work or school due to illness or intervention generated by our ABM fed into FluEcon, our Monte Carlo economic simulation model, which was developed in microsoft excel (Microsoft, Redmond, WA) with the crystal ball add-in (Oracle, Redwood City, CA), to translate influenza cases into direct health care costs and indirect costs (i.e., lost productivity from illness and mortality) as detailed in previous publications (Brown et al. 2011; Lee et al. 2012a, 2012b, 2015). Each symptomatic individual had probabilities of seeking outpatient treatment, being hospitalized, or dying from influenza (Molinari et al. 2007). Each of these outcomes were age-specific and were associated with a corresponding cost; thus, the outcome of each case determined the costs accrued. Outpatient visit and hospitalization costs came from nationally 
representative databases (Centers for Medicare and Medicaid Services 2011; Thomson Healthcare 2008 U.S. Department of Health and Human Services 2011).

Symptomatic cases resulted in lost productivity during the duration of their symptoms (if not hospitalized) or hospitalization. The median hourly wage for all occupations from the Department of Labor served as proxies for productivity losses and assumed an 8-h workday (U.S. Bureau of Labor Statistics 2010). Agents that died incurred productivity losses representing the net present value of that person's remaining lifetime earnings, based on their life expectancy as derived from the human mortality database (University of California, Berkeley and Max Planck Institute for Demographic Research 2011). A $3 \%$ discount rate converted all costs into 2014 values.

Total influenza-related costs included direct and indirect costs, including missed work, school, and sick days due to influenza. For intervention costs, we assumed that all adults missing work due to closure incurred a day of lost productivity and there was no possibility of telecommuting. Because businesses and schools were closed on the same day, we assumed a child missing school would have a parent at home or would be cared for by a neighbor or relative, resulting in no additional productivity losses.

Mitchell County, North Carolina residents reported this experience responding to a 2-week school closure brought on by an influenza B epidemic in fall 2006. According to a study, "For parents, the outbreak and school closings meant missing work, getting relatives to watch children, even taking well youngsters to work" (White and Young 2006, FluTrackers.com).

\subsection{Varying weekend length}

The set of experiments on weekends had two objectives. The first was to determine whether including weekends makes a difference in simulation models of infectious disease transmissions. The second was to assess if extending the weekend from 2 to 3 days can mitigate the severity of an epidemic.

Every day, all agents, including students, potentially interact with each other in the community, although with a fairly low probability of transmitting the virus. On weekends, student agents do not go to school but have an undetermined number of community interactions. Our model assumed that students would have (1) no extra weekend contacts, (2) $25 \%$ more community weekend contacts than community weekday contacts, or (3) $100 \%$ more community weekend contacts than community weekday contacts. Assumption 2 is more consistent with estimates derived from information on French student vacationers (Cauchemez et al. 2008).

\subsection{Simulation scenarios}

We used several simulation scenarios to produce comparisons to investigate the study objectives. For all scenarios, the effect of prior immunity to the H1N1 pathogen protects older adults but not school-aged children (Miller et al. 2010). We present and compare six specific scenarios in Table 3. Scenario WE-0 assumes every day of the week has the same contact profile, similar to assumptions in many models. 
Table 3 Summary of simulated scenarios

\begin{tabular}{ll}
\hline Scenario-id & Description \\
\hline WE-0 & No weekends \\
WE-2a & 2-day weekend-no compensatory behavior \\
WE-2b & 2-day weekend-baseline compensatory behavior response \\
WE-2c & 2-day weekend-baseline compensatory behavior response times 2 \\
WE-3a & 3-day weekend triggered after $100 \mathrm{~K}$ infections. 3-day weekend remains \\
& to the end of the run \\
WE-3b & 3-day weekend triggered after $100 \mathrm{~K}$ infections. 3-day remains for \\
& 60 days then reverts to 2-day weekend \\
\hline
\end{tabular}

We used three 2-day weekend scenarios. Scenario WE-2a assumes that on weekdays children interact with other children and adults at malls, various shopping venues, and social events. On weekends, these children stay home and only interact within the household. This unlikely scenario would be a strict quarantine on weekends. Scenario WE-2b assumes that children interact with other students and adults on weekdays and on weekend days students increase their weekday contacts by $25 \%$. These estimates are based on a French study that assumes that during holidays, transmission does not occur in schools but in other places such as the household and the community (Cauchemez et al. 2009). We assume that the change in patterns of contact during holidays is similar to changes between weekdays and weekends. The increase suggested by the French study was a $50 \%$ larger contact rate during holidays. We assume a smaller $25 \%$ increase per day (for 2 days). Finally, scenario WE-2c increases the weekend contacts by $100 \%$ above the baseline-beyond what we believe to be a realistic assumption, but one that supports a simple sensitivity analysis by bracketing the "true" compensatory contact behavior pattern.

The other two scenarios assume 3-day weekends. Scenario WE-3a simulates a 3-day weekend run that assumes that after a specific threshold number of cases is reached (100,000 cases was selected arbitrarily), school operations then move to a 4-day school attendance week and that this pattern extended to the end of the epidemic period. Scenario WE-3b used the same threshold (100,000 cases) but only operates this policy for 60 days. During the extended weekend period, there is no difference in contact patterns except on that extra weekend day. For Scenarios WE$3 \mathrm{a}$ and WE-3b, on the third weekend day there are no school contacts but there is a $25 \%$ increase in community contacts.

\section{Results}

Figures 1 through 4 and Table 4 describe the comparisons among the scenarios listed in Table 3.

As shown in Fig. 1, both the WE-2a and WE-0 scenarios display the infection curve of total infections by day and each scenario is calibrated to the same criteria (Table 2) with an illness attack rate of $16 \%$. The scenarios without a weekend 


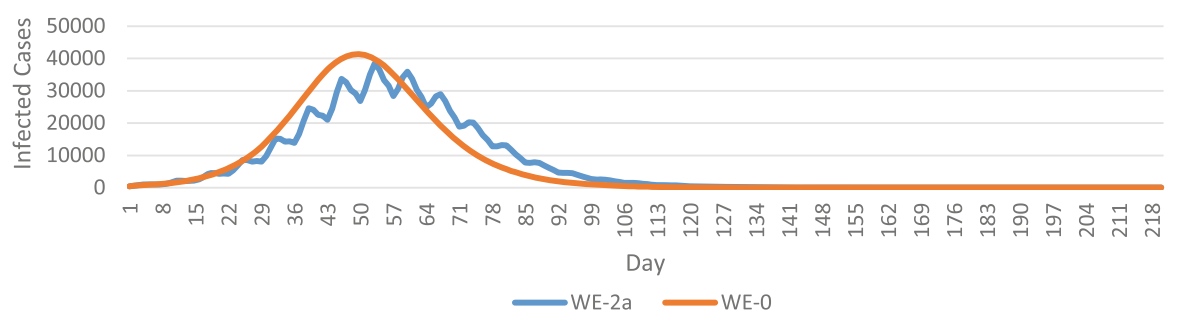

Fig. 1 Comparison between 2-day weekend (WE-2a) and 0-day weekend (WE-0) models

(WE-0) are smoother and are left-shifted, in contrast to the 2-day weekend without compensatory behavior scenario (WE-2a). Also, the no-weekend model has a shorter epidemic period (from 175 to 168 days), has a similar maximum peak daily infection (41,348 versus 38,407), and an earlier peak case incidence day (49 vs. 54).

The four scenarios shown in Fig. 2 are calibrated to the criteria defined in Table 2 and accordingly the area under each curve records nearly identical infection prevalence. The 2-day weekend scenarios with varying rates of compensatory behavior are all right-shifted relative to the no-weekend scenario (WE-0). The scenario with a $100 \%$ increase of compensatory contacts on weekends (WE-2c) approximates most closely the shape of the no-weekend scenario attack rate curve. Because the results of other weekend studies support a lower rate of compensatory behavior, we felt that adding an average of $25 \%$ increases in compensatory community contacts of students on weekends was the most realistic with respect to the supporting data. We characterize this scenario as the baseline scenario (WE-2b).

Figure 3 compares a 2-day weekend baseline scenario (WE-2b) with a 3-day weekend scenario that operates for only 60 days (WE-3b). The WE-2b curve shows that initiating an extended 3-day weekend on day 28 of the epidemic (after reaching 100,000 cases), has a noticeable effect on the peak incidence rate and the overall attack rate. Maintaining a 3-day weekend policy for an arbitrary 60-day period substantially reduces (by more than half) both the peak and overall serological attack rate (24.0-16.5\%). This demonstrates the potential benefit of the 3-day weekend as a containment strategy in contrast to full school closure, but leaves the cost issue unresolved.

Figure 4 shows a comparison of both 3-day weekend scenarios. WE-3a initiates the 3-day weekend on day 28 and sustains it to the end of the epidemic period; WE$3 \mathrm{~b}$ also initiates the 3-day weekend on day 28 but sustains it for 60 days before reverting back to a 2 -day weekend. Figure 4 suggests there is little difference between the two scenarios and suggests that to be cost-effective, identifying a second threshold that causes the 3-day weekend intervention to revert to the status quo is important.

To demonstrate the economic difference between the two scenarios, we performed an economic analysis of the two 3-day weekend scenarios in contrast to the baseline no-intervention scenario (WE-2b) and the results are shown in Table 4. Based on the model assumptions reported, the mean estimated savings to society for implementing a 3-day weekend intervention in a Chicago-like city ranges from $\$ 332.9$ million to $\$ 248.6$ million. In other words, closing schools for 9 


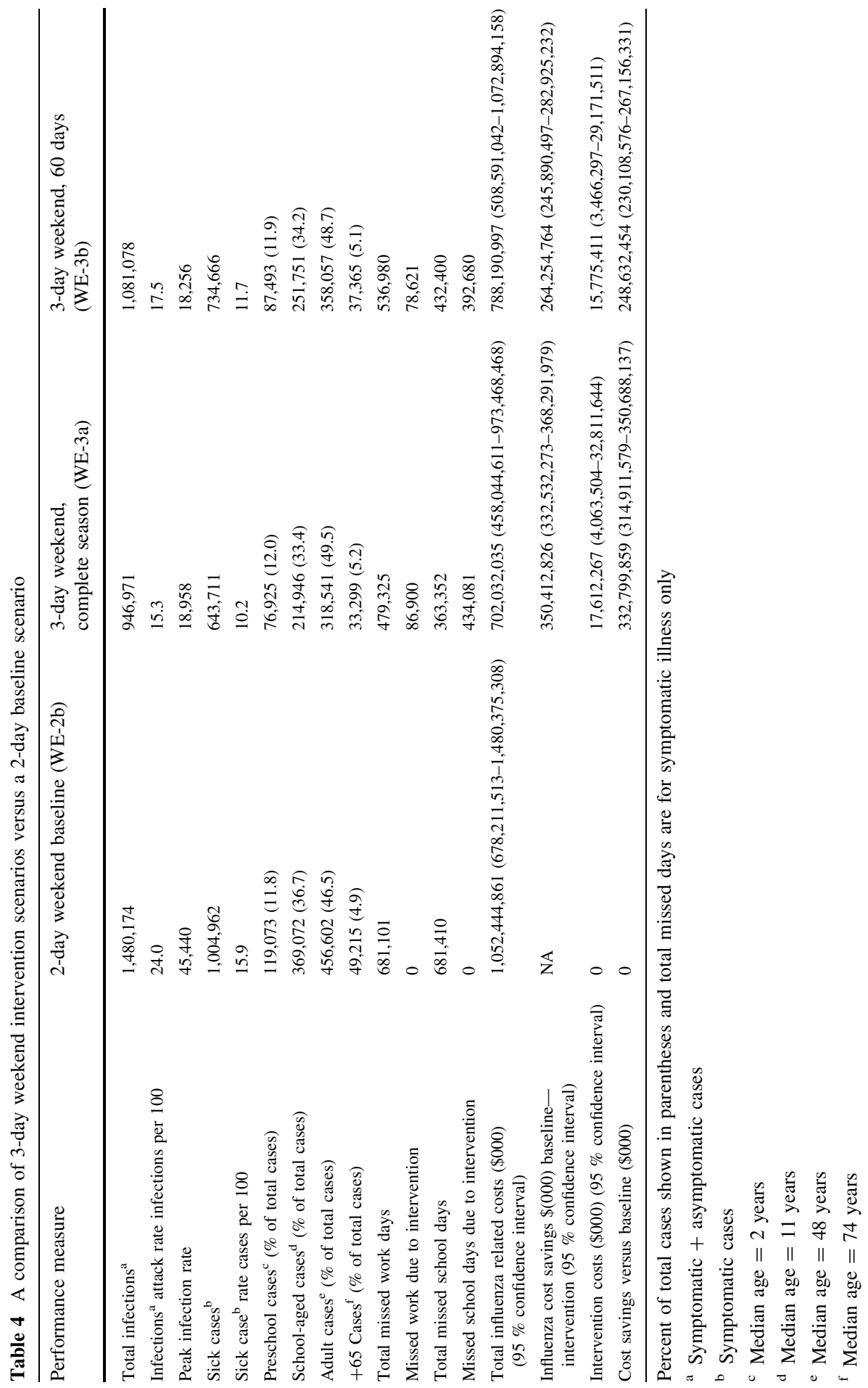




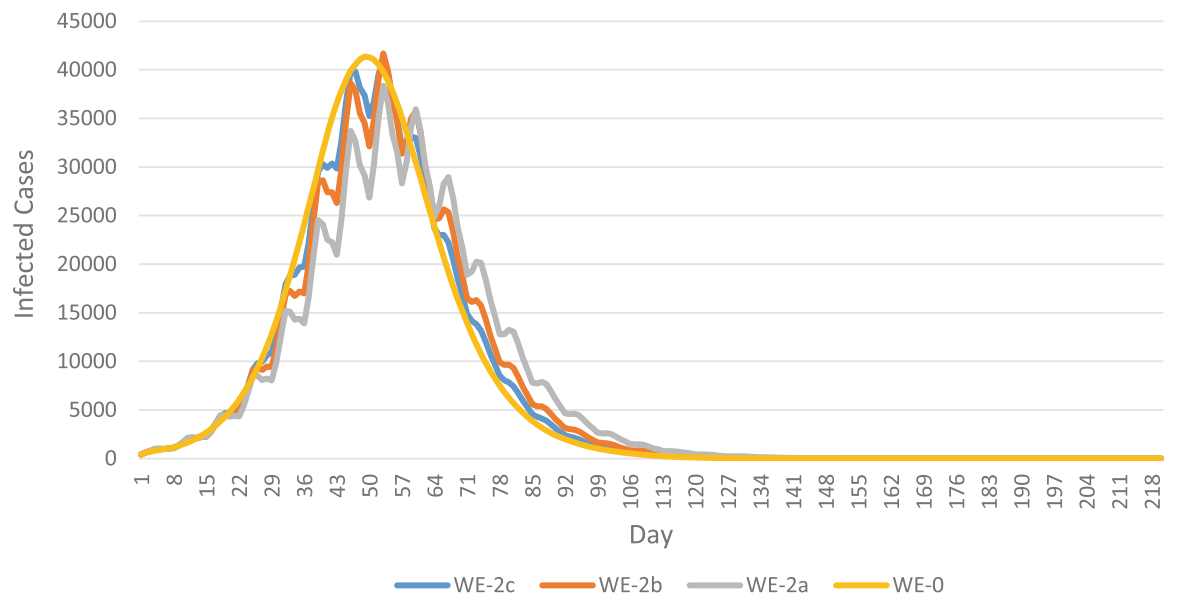

Fig. 2 Compensatory behavior analysis

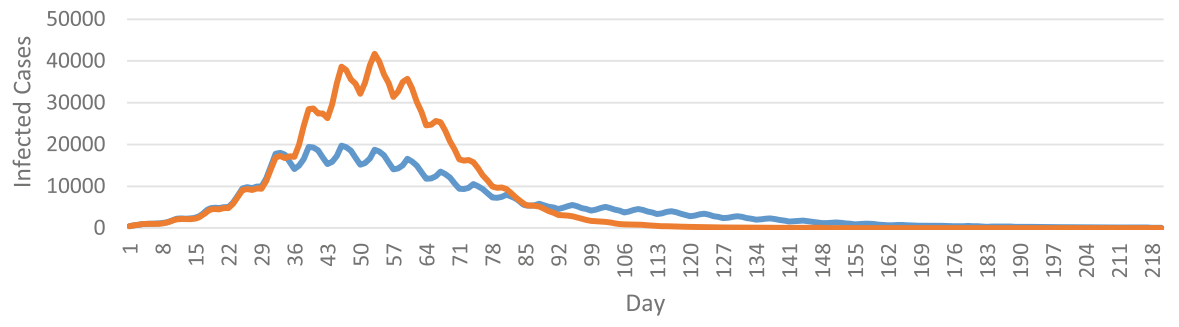

$\longrightarrow$ WE-3b WE-2b

Fig. 3 Effect of adding a third day to the weekend

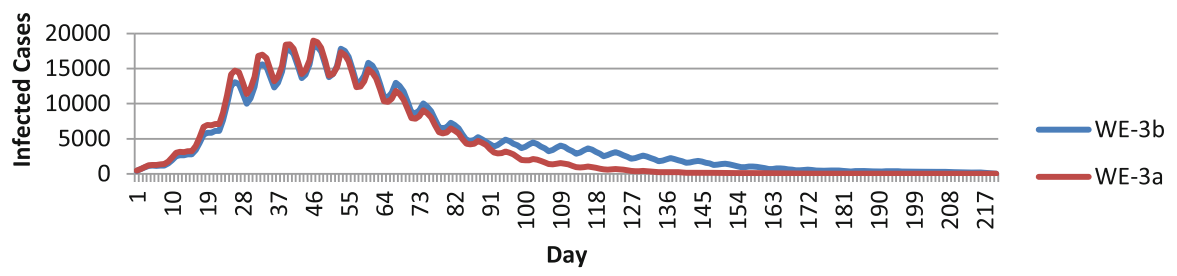

Fig. 4 Effects of ending the 3-day weekend at different points in the epidemic

Mondays during the peak period of a flu pandemic in a city similar to Chicago could possibly prevent up to $\$ 248.6$ million in losses. The range of the estimates is based on the length of the intervention and do not reflect any differences in the educational benefit attributed to a 5- versus a 4-day work/school week. These cost estimates in Table 4 include mean and 95 confidence intervals for the cost estimates generated by FluEcon. The standard errors due to the Monte Carlo process are small resulting in small 95 confidence intervals and are not shown. 


\section{Discussion}

Using an epidemic model that has been calibrated to reproduce the patterns of the H1N1 epidemic of 2009, this study assessed the implications of representing weekend patterns in influenza models. The results indicate that leaving weekend behaviors out of a model shortens the predicted epidemic period unless unrealistically high rates of compensatory community contacts (with school friends, mall encounters, etc.) are also represented. With the exception of higher school infection rates and a leftward shift in the epidemic, there is little difference between the 0 weekend and the 2-day weekend models. This result is directly attributed to a high rate of withdrawal $(90 \%)$ to the home by symptomatic individuals.

Extending the weekend from 2 to 3 days was simulated to assess if 3-day weekends were an effective influenza intervention strategy. The results suggest that extended weekends could have a significant effect on reducing peak attack rates and societal costs. It is also possible that reducing peak attack rates could extend the epidemic period. Although the model does not incorporate seasonal effects, it may overstate the overall infection rate, which would diminish as a consequence of seasonal effects, especially during the later stages of the epidemic period.

Overall, the evidence from our simulations suggests that using a 3-day weekend as an intervention strategy could be effective for mild epidemics similar in severity to the H1N1 epidemic of 2009 and with a concentration in schoolaged children. Such an intervention would also be far less detrimental to the educational process than sustained closure because students and teachers could maintain contact throughout the epidemic period. In addition, a 4-day week might easily be accommodated by many types of businesses and schools; in fact, 21 states currently support 4-day school weeks. (National Conference of State Legislators 2013). There certainly could be substantial costs to closing schools for this additional day, especially in urban areas. Two separate studies reported that closing schools completely for a sustained period during the 2009 H1N1 epidemic could have resulted in substantial societal costs because the lost productivity and child care costs could have far outweighed the savings created by preventing influenza cases (Brown et al. 2011; Lempel et al. 2009). However, our economic analysis suggests the opposite would occur if schools are closed only 1 day per week for a 2-month period. The lower costs and subsequent savings associated with nine consecutive 3-day weekends during the critical 2-month period of the H1N1 influenza season would more than compensate the costs of school and workplace closure.

Acknowledgments This work was supported by the Agency for Healthcare Research and Quality (AHRQ) via grant R01HS023317 and the National Institute of Child Health and Human Development (NICHD) and the Global Obesity Prevention Center (GOPC) via grant U54HD070725. The funders had no role in the design and conduct of the study; collection, management, analysis, and interpretation of the data; and preparation, review, or approval of the manuscript. The authors thank Craig R. Hollingsworth for technical writing and editing assistance. 


\section{References}

Albright KC, Raman R, Ernstrom K, Hallevi H, Martin-Schild S, Meyer DM, Meyer BC, Morales MM, Grotta JC, Lyden PD, Savitz SI (2009) Can comprehensive stroke centers erase the 'weekend effect'? Cerebrovasc Dis 27:107-413

Araz OM, Damien P, Paltiel DA, Burke S, van de Geijn B, Galvani A, Meyers LA (2012) Simulating school closure policies for cost effective epidemic decision making. BMC Public Health 12:449. doi:10.1186/1471-2458-12-449

Atkinson-Palombo CM, Miller JA, Balling RC Jr (2006) Quantifying the ozone "weekend effect" at various locations in Phoenix, Arizona. Atmos Environ 40:7644-7658

Beckman RJ, Baggerly KA, McKay MD (1996) Creating synthetic baseline populations. Transp Res Part A 30:415-429

Bonne J (2003) Are we done with the 40-hour week? How technology, productivity and family change the way we work. Bus Spec Rep. http://www.msnbc.msn.com/id/3072426. Accessed Dec 15, 2009

Brown ST, Tai JH, Bailey RR, McGlone SM, Cooley PC, Wheaton WD et al (2011) Would school closure for the $2009 \mathrm{H} 1 \mathrm{~N} 1$ influenza epidemic have been worth the cost?: a computational simulation of Pennsylvania. BMC Public Health 11:353

Cajka J, Cooley PC, Wheaton WD, Ganapathi L, Wagener D (2010) Attribute assignment to a synthetic population in support of agent-based disease modeling (RTI Press Publication). Research Triangle Park, NC: RTI International. http://www.rti.org/pubs/mr-0019-1009-cajka.pdf

Cauchemez S, Valleron AJ, Boëlle PY, Flahault A, Ferguson NM (2008) Estimating the impact of school closure on influenza transmission from sentinel data. Nature 452(7188):750-754

Cauchemez SA, Bhattarai A, Marchbanks TL, Fagan RP, Ostroff S, Ferguson NM (2009) Role of social networks in shaping disease transmission during a community outbreak of H1N1 2009 pandemic influenza. PNAS 108:2825-2830. doi:10.1073/pnas.1008895108

Centers for Disease Control and Prevention (2009) 2009 H1N1 Early Outbreak and Disease Characteristics. Historical Archive. http://www.cdc.gov/h1n1flu/surveillanceqa.htm\#5, Accessed on Oct 27, 2009

Centers for Medicare and Medicaid Services (2011) Physicians fee schedule. U.S. Department of Health and Human Services. Baltimore, MD. http://www.cms.hhs.gov/, Accessed on Accessed 16 March 2011

Chen H, Singal V (2003) Role of speculative short sales in price formation: the case of the Weekend Effect. J Financ 58:685-706

Cooley PC, Lee BY, Brown S, Cajka J, Chasteen B, Ganapathi L et al (2010) Protecting health care workers: a pandemic simulation based on Allegheny County. Influ Other Respir Viruses 4:61-72

Cooley PC, Brown ST, Cajka J, Chasteen B, Ganapathi L, Grefenstette J, Hollingsworth CR, Lee BY, Levine B, Wheaton WD, Wagener DK (2011) The role of subway travel in an influenza epidemic: a New York City simulation. J. Urban Health 88:982-995. doi:10.1007/s11524-011-9603-4

Eames KT, Tilston NL, White PJ et al (2010) The impact of illness and the impact of school closure on social contact patterns. Health Technol Assess 14:267-312

Eames KT, Tilston NL, Edmunds WJ (2011) The impact of school holidays on the social mixing patterns of school children. Epidemics 3(2):103-108

Eames KT, Tilston NL, Brooks-Pollock E et al (2012) Measured dynamic social contact patterns explain the spread of H1N1 influenza. PLoS Comput Biol 8(3):e1002425

Edmunds W, Kafatos G, Wallinga J, Mossong J (2006) Mixing patterns and the spread of close-contact infectious diseases. Emerg Themes Epidemiol 3:10

Ferguson N, Cummings D, Cauchemez S et al (2005) Strategies for containing an emerging influenza epidemic in Southeast Asia. Nature 437:209-214

Ferguson NM, Cummings DA, Fraser C, Cajka JC, Cooley PC, Burke DS (2006) Strategies for mitigating an influenza epidemic. Nature 442:448-452

Germann T, Kadau K, Longini IJ, Macken C (2006) Mitigation strategies for epidemic influenza in the United States. PNAS 103:5935-5940. doi:10.1073/pnas.0601266103

Halloran EM, Eubank S, Ferguson MN, Longini MI, Barrett C, Beckman R, Burke SD, Cummings AD, Fraser C, Germann CT, Kadau K, Lewis B, Macken AC, Vullikanti A, Wagener DK, Cooley PC (2008) Modeling targeted layered containment of an influenza epidemic in the USA. Proc Natl Acad Sci 105:4639-4644 
Hens N, Ayele GM, Goeyvaerts N et al (2009) Estimating the impact of school closure on social mixing behaviour and the transmission of close contact infections in eight European countries. BMC Infect Dis 9:187

Lee BY, Brown ST, Cooley PC, Potter MA, Wheaton WD, Voorhees RT et al (2010a) Simulating school closure strategies to mitigate an influenza epidemic. J Public Health Manag Pract 16:252-261

Lee BY, Brown ST, Korch G, Cooley PC, Zimmerman RK, Wheaton WD et al (2010b) A computer simulation of vaccine prioritization, allocation, and rationing during the 2009 H1N1 influenza epidemic. Vaccine 12:4875-4879

Lee BY, Brown ST, Cooley PC, Zimmerman RK, Wheaton WD, Zimmer SM et al (2010c) A computer simulation of employee vaccination to mitigate an influenza epidemic. Am J Prev Med 38:247-257

Lee BY, Bartsch SM, Willig AM (2012a) The economic value of a quadrivalent versus trivalent influenza vaccine. Vaccine 30:7443-7446

Lee BY, Tai JHY, McGlone SM et al (2012b) The potential economic value of a "universal" (multi-year) influenza vaccine. Influ Other Respir Viruses 6:167-175

Lee BY, Bartsch SM, Brown ST, Cooley PC, Wheaton WD, Zimmerman RK (2015) Quantifying the economic value and quality of life: Impact of earlier influenza vaccination. Medical Care 53(3):218-229

Lempel H, Epstein JM, Hammond RA (2009) Economic cost and health care workforce effects of school closures in the U.S. PLoS Curr, 1 RRN1051. doi: 10.1371/currents.RRN1051

Longini IJ, Nizam A, Xu S et al (2005) Containing epidemic influenza at the source. Science 309:1083-1087

Mao L (2011) Agent-based simulation for weekend-extension strategies to mitigate influenza outbreaks. BMC Public 11:522. doi:10.1186/1471-2458-11-522

Marr LC, Harley RA (2002) Modeling the effect of weekday-weekend differences in motor vehicle emissions on photochemical air pollution in central California. Environ Sci Technol 36:4099-4106. doi:10.1021/es020629x

McCaw J, Forbes K, Nathan P, Pattison P, Robins G, Nolan T, McVernon J (2010) Comparison of three methods for ascertainment of contact information relevant to respiratory pathogen transmission in encounter networks. BMC Infect Dis 10:166

Miller E, Hoschler K, Hardedid P, Stanford E, Andrews N, Zambon M (2010) Incidence of 2009 pandemic influenza in England: a cross sectional serological study. Lancet 375:1100-1108. doi:10. 1016/S0140-6736(09)62126-7

Molinari N-AM, Ortega-Sanchez IR, Messonnier ML et al (2007) The annual impact of seasonal influenza in the US: measuring disease burden and costs. Vaccine 25:5086-5096

National Conference of State Legislators (2013) Four-day school weeks. National conference of state legislators. http://www.ncsl.org/research/education/school-calendar-four-day-school-week-overview. aspx. Accessed Oct 2013

Riley S, Kwok KO, Wu KM, Ning DY, Cowling BJ et al (2011) Epidemiological characteristics of 2009 (H1N1) pandemic influenza based on paired sera from a longitudinal community cohort study. PLoS Med 8(6):e1000442. doi:10.1371/journal.pmed.1000442

Rothberg MB, Rose DN (2005) Vaccination versus treatment of influenza in working adults: a costeffectiveness analysis. Am J Med 118:68-77

Spiers PS, Guntheroth WG (1999) The effect of the weekend on the risk of sudden infant death syndrome. Pediatrics 104(5):e58

Thomson Healthcare (2008) MarketScan research database. Thomson Healthcare, Ann Arbor

University of California, Berkeley and Max Planck Institute for Demographic Research (2011) Human mortality database. http://www.mortality.org, Accessed 14 June 2011

U.S. Census Bureau (2009) American fact finder. http://factfinder.census.gov/home/saff/main. Accessed 18 Feb 2009

U.S. Bureau of Labor Statistics (2010) Occupational employment statistics: May 2009 national occupational employment and wage estimates, United States. Washington, DC. http://stat.bls.gov/ oes/2008/may/oes_nat.htm\#b00-0000. Accessed Nov 2011

U.S Bureau of Labor Statistics (2013) Civilian labor force participation rates by age, sex, race, and ethnicity. http://www.bls.gov/emp/ep_table_303.htm

U.S. Bureau of Labor Statistics (2014) American time use survey summary. http://www.bls.gov/news. release/atus.nr0.htm

U.S. Department of Health and Human Services (2011) HCUP facts and figures: Statistics on hospitalbased care in the United States. Agency for Healthcare Research and Quality, Rockville, MD. http:// hcupnet.ahrq.gov/HCUPnet.jsp. Accessed 4 Sep 2013 
Van Kerckhove K, Hens N, Edmunds WJ, Eames KTD (2013) The impact of illness on social networks: implications for transmission and control of influenza. Am J Epidemiol 178:1655-1662. doi:10. 1093/aje/kwt196

Wheaton WD, Cajka JC, Chasteen BM, Wagener DK, Cooley PC, Ganapathi L (2009) Synthesized population databases: A U.S. geospatial database for agent-based models. RTI Press, Research Triangle Park, NC. http://www.rti.org/pubs/mr-0010-0905-wheaton.pdf

White G, Young A (2006) CDC puts rapid N.C. flu outbreak under microscope. http://www.flutrackers. com/forum/showthread.php?t=12748. Accessed Oct 2013

Writing Committee of the WHO Consultation on Clinical Aspects of Epidemic (H1N1) (2009) Influenza (2010) Clinical aspects of epidemic 2009 influenza A (H1N1) virus infection. N Engl J Med 362:1708-1719

Philip C. Cooley MSc, is a Senior RTI Fellow in bioinformatics and high-performance computing and principal scientist and Assistant Director of Bioinformatics. He has more than 40 years of experience developing computer models to study environmental health and disease transmission scenarios. He has been extensively involved in designing and implementing influenza transmission models to study and manage pandemic influenza and he is currently the Co-PI of the MIDAS Informatics Group. His current research includes an assessment of statistical methods for biomarker explorations as part of genome-wideanalysis studies, and to support this effort he has developed a synthetic database of loci with known genetic properties.

Sarah Bartsch MSc, is the Research Coordinator and a Senior Research Analyst with the Public Health Operations and Research (PHICOR) Group with Johns Hopkins University (formerly at the University of Pittsburgh). She has a Bachelor of Science in Biology with a minor in Chemistry from Franciscan University of Steubenville (2007) and a Master's in Public Health in Epidemiology from The Ohio State University (2009). Her research primarily focuses on economic modeling of various health care associated infections (HAIs), the effects of patient sharing among hospital networks, neglected tropical diseases, and malaria.

Shawn T. Brown Ph.D, is the Director of Public Health Applications at the Pittsburgh Supercomputing Center (PSC). He is the Technical Lead of the HERMES Supply Chain Logistics Team (http://hermes. psc.edu) and lead of the NIH MIDAS Network Software Working Group. His current research interests are in using simulations for informed decision support, vaccine supply chains in developing nations, agent-based modeling of disease dynamics and the use of high-performance computing in simulation and modeling. His Public Health Applications Group at the PSC leads the Bill and Melinda Gates Funded VecNet Cyber infrastructure Project for malaria modeling and he is a Co-Investigator for the National Institute of Health (NIH) Modeling of Infectious Diseases Agent Study (MIDAS) and Co-Principle Investigator on the MIDAS Information Technology Resource at RTI International. Dr. Brown received his $\mathrm{PhD}$ in Quantum Chemistry at the University of Georgia and his B.S. in Chemistry at Bethany College, WV.

William D. Wheaton MA, is the director of the Geospatial Science and Technology Program and a senior research geographer at RTI International. He has 27 years of experience with applying geographic information systems (GIS) technology in environmental and social science research. As director of this program, he leads and coordinates GIS tasks in support of diverse research throughout RTI and for external government and commercial clients. In addition to maintaining technical proficiency with GIS and related technologies, Mr. Wheaton has an extensive background teaching GIS and related topics to industry and government. He is the task lead on the Data Identification and Acquisition task on the Models of Infectious Disease Agent Study (MIDAS). His current recent interests have focused on the development of realistic synthetic populations to support simulations using Agent-Based Model.

Diane K. Wagener Ph.D, is a senior epidemiologist in the Genomics, Statistical Genetics and Environmental Research Program in RTI International's Statistics and Epidemiology unit. Dr. Wagener has more than 35 years of experience in academic and governmental institutions. At RTI, she currently directs projects of national biosecurity importance. She was Chief of the Environmental Studies Branch at 
CDC and Senior Project Officer in the Board on Environmental Studies at the National Academy of Sciences. In academia and in the government, Dr. Wagener has conducted studies of insulin-dependent diabetes, asthma, breast cancer, brain cancer, alpha-1-antitrypsin deficiency, depression, schizophrenia, and leprosy. She received a National Institute of Mental Health Research Scientist Development Award to study gene and environment interactions in major mental diseases. Her research also includes methodologic studies of statistical issues, such as summary measures of population health and predictive value of genetic risk factors.

Bruce Y. Lee MD, MBA is Associate Professor of International Health at the Johns Hopkins Bloomberg School of Public Health and Director, Operations Research, at the International Vaccine Access Center. His group specializes in developing economic, and operational computer models that help decision makers tackle infectious diseases of global importance. He is the Scientific Lead for the HERMES Project and the RHEA Project. His funding has included grants from the Bill and Melinda Gates Foundation, the National Institutes of Health (NIH), Centers for Disease Control and Prevention (CDC), National Science Foundation (NSF), National Library of Medicine (NLM), and the Pennsylvania Department of Health. During the 2009 H1N1 influenza pandemic, he was embedded in the Department of Health and Human Services, helping with the national response. 